\title{
Acute pulmonary embolism in hospitalized patients with SARS-CoV-2-related pneumonia: multicentric experience from Italian endemic area
}

\author{
Davide Ippolito ${ }^{1,2} \cdot$ Teresa Giandola $^{1,2} \cdot$ Cesare Maino ${ }^{1,2}$ - Anna Pecorelli ${ }^{1,2} \cdot$ Carlo Capodaglio $^{1,2} \cdot$ Maria Ragusi $^{1,2}$. \\ Marco Porta ${ }^{1,2} \cdot$ Davide Gandola $^{1,2} \cdot$ Alessandro Masetto $^{3} \cdot$ Silvia Drago $^{2} \cdot$ Pietro Allegranza $^{4} \cdot$ Rocco Corso $^{2}$. \\ Cammillo Talei Franzesi ${ }^{1,2} \cdot$ Sandro Sironi ${ }^{1,5}$
}

Received: 26 August 2020 / Accepted: 14 December 2020 / Published online: 8 January 2021

(c) Italian Society of Medical Radiology 2021

\begin{abstract}
Purpose To analyze pulmonary embolism (PE) on chest computed tomography pulmonary angiography (CTPA) in hospitalized patients affected by SARS-CoV-2, according to the severity of lung disease based both on temporal CT features changes and on CT-severity lung involvement (CT-severity score), along with the support of clinical and laboratory findings. Methods We retrospectively enrolled a total of 170 patients with confirmed SARS-CoV-2 infection who underwent CTPA examination for PE suspicion. Pulmonary arteries diameters, right ventricle/left ventricle (RV/LV) ratio, presence, absence, and distribution of PE, pulmonary artery obstructive index (PAO index), and lobe involvement were recorded. All CT scans were reviewed to assess temporal CT changes and the COVID CT-severity score.

Results A total of 76 out of 170 patients (44.7\%) developed PE without having any major risk factors for venous thromboembolism. The most severe pulmonary arteries involvement, expressed in terms of PAO Index, occurred in those patients with markedly elevated D-dimer and C-reactive protein (CRP) values and those patients with an advanced temporal stage of lung disease. The majority PE-positive patients were hospitalized in non-intensive wards. PE-positive patients showed a slightly higher hospitalization time in comparison with PE-negative ones. In the three months of study, overall $85.9 \%$ of patients were discharged while $14.1 \%$ died, of whom 13 PE-positive $(54.2 \%)$.

Conclusions Patients hospitalized for SARS-CoV-2 infection present a higher cumulative incidence of PE compared to the general population of hospitalized patients, regardless of the severity of lung inflammation or the temporal stage of the disease.
\end{abstract}

Keywords Infections $\cdot$ Coronavirus $\cdot$ Tomography $\cdot$ X-ray computed $\cdot$ Acute pulmonary embolism

\begin{tabular}{|c|c|c|c|}
\hline \multicolumn{2}{|c|}{ Abbreviations } & \multirow[t]{2}{*}{ СТРA } & \multirow{2}{*}{$\begin{array}{l}\text { Computed tomography pulmonary } \\
\text { angiography }\end{array}$} \\
\hline ATIII & Antithrombin III & & \\
\hline \multirow[t]{3}{*}{ CT-SS } & CT-severity score & ET & Endotracheal tube \\
\hline & & GGO & Ground-glass opacity \\
\hline & & MPR & Multiplanar reconstructions \\
\hline Suppl & & $\begin{array}{l}\text { NIV } \\
\text { PAO index }\end{array}$ & $\begin{array}{l}\text { Noninvasive ventilation } \\
\text { Pulmonary arterv obstructive index }\end{array}$ \\
\hline
\end{tabular}
s11547-020-01328-2).

Cesare Maino

mainocesare@gmail.com

1 Department of Diagnostic Radiology, School of Medicine, University of Milano-Bicocca, H. S. Gerardo, Via Pergolesi 33, 20900 Monza, Milan, Italy

2 Department of Diagnostic Radiology, University of Milano-Bicocca, H. S. Gerardo, Monza, Italy
3 Department of Diagnostic Radiology, Ospedale Vimercate, Vimercate, Italy

4 Department of Diagnostic Radiology, Desio Hospital, Monza, Italy

5 Department of Diagnostic Radiology, Papa Giovanni XXIII Hospital Bergamo, Bergamo, Italy 
PE Pulmonary embolism

$\mathrm{RV} / \mathrm{LV}$ ratio Right ventricle/left ventricle ratio

\section{Introduction}

Since December 2019, several cases of viral pneumonia caused by a novel coronavirus (COVID-19), identified as severe acute respiratory syndrome coronavirus 2 (SARSCoV-2), have been reported worldwide after the initial outbreak in Wuhan, China.

As of August 26, 2020, there were about 24 million confirmed cases of SARS-CoV-2 all over the world and 820,000 patients died with a mortality rate of $5.1 \%$. Due to the high contagious rate, laboratory detection has become time-consuming and it may not be available for all people with suspected infection owing to the shortage of test kits for SARS-CoV-2. According to the current experience, pulmonary findings can be detected by imaging techniques in the initial phase of infection, earlier than clinical symptoms, and therefore imaging plays an important role in the preclinical screening, primary diagnosis, and management of SARS-CoV-2-related pneumonia [1-4].

According to Fleischner Society guidelines and European Society of Radiology advice paper, computed tomography $(\mathrm{CT})$ can be considered a useful tool in patients with respiratory symptoms and not as a screening $[5,6]$.

CT features of SARS-CoV-2-related pneumonia have now been extensively described [7-10] and as reported in the recent literature, coagulation disorders are common and thromboembolic complications are increasingly being reported [11-14]. In this new SARS-CoV-2 infection, recent studies have shown a higher risk of developing thrombotic events [11-13], emphasizing the need to evaluate the D-Dimer value and other coagulation tests during the management, to regularly perform risk assessment. Abnormal coagulation results, especially markedly elevated D-dimer is associated with poor prognosis [15]. Infection, immobilization, respiratory failure, and mechanical ventilation are further considered potential thromboembolic-inducing factors in SARS-CoV-2 patients. Moreover, they might also be prone to develop venous and arterial thromboembolic disease due to excessive inflammation, hypoxia, and disseminated intravascular coagulation (DIC) [16-19].

On these bases, this study aims to analyze the pulmonary embolism detected on computed tomography pulmonary angiography (CTPA) in hospitalized patients affected by SARS-CoV-2, according to the disease severity based both on temporal CT features changes and on semiquantitative CT-severity lung involvement, along with the support of clinical and laboratory findings.

\section{Methods}

The study was performed under the Declaration of Helsinki; clinical and radiological data was anonymized during data collection. Given the retrospective nature of the study and in the presence of technical difficulties during the pandemic, informed consent will be waived, following article 89 of the GDPR EU Regulation 2016/679.

\section{Patient population}

From March 4th, 2020 until April 24th, 2020 we retrospectively enrolled all hospitalized patients with SARS-CoV-2 infection, confirmed through a pan-coronavirus conventional polymerase chain reaction (PCR) assay.

Data were extracted from the electronic medical records database of three different hospitals and tertiary care referral centers in Lombardy, a Northern Italian region.

Inclusion criteria were: (1) PCR-confirmed diagnosis of SARS-CoV-2 infection, (2) laboratory test, including D-dimer value, white blood cell (WBC) differential, C-reactive protein (CRP) values, (3) complete arterial blood gas (ABG) test. The exclusion criterion was the presence of severe breathing-induced artifacts on the CTPA scan.

All patients with clinical suspicion of PE including chest pain, worsening of respiratory symptoms, irregular or new-onset rapid heartbeat, worsening of fever, aggravation of ABG parameters, and a marked increase over time of D-dimer (normal range 262-62.000 ng/ml) and/or fibrinogen values (normal range $246-983 \mathrm{mg} / \mathrm{dl}$ ) despite the anticoagulant therapy, were addressed to CTPA.

\section{Patients characteristics}

Patient history and major predisposing risk factors, including coagulation disorders, oral contraceptives, recent surgical procedures, pregnancy, cancer, fractures of the lower limb, and previous deep vein thrombosis, were recorded as well.

According to the guidelines implemented by the hospitals taking part in the study, patients with D-dimer score over $5000 \mu \mathrm{g} / \mathrm{mL}$ underwent a complete anticoagulation therapy (Enoxaparin sodium, $100 \mathrm{UI} / \mathrm{kg}$ twice a day), while patients with D-dimer score under $5000 \mu \mathrm{g} / \mathrm{mL}$ underwent prophylactic anticoagulation (Enoxaparin sodium, $100 \mathrm{UI} / \mathrm{kg}$, once a day).

\section{Laboratory findings, ventilation, and outcome}

For each patient, we collected the following laboratory test results, performed on the same day of the CTPA: (1) 
D-dimer value, (2) white blood cell count (WBC), (3) neutrophils, (4) lymphocytes, (5) platelets (PLT), (6) CRP value, (7) fibrinogen, (8) antithrombin III (ATIII), and (9) international normalized ratio (INR). For each patient, ABG data, including $\mathrm{pO}_{2}, \mathrm{pCO}_{2}, \mathrm{HCO}_{3}, \mathrm{pH}, \mathrm{SaO}_{2}$, was recorded. $\mathrm{P} / \mathrm{F}$ ratio, expressing the ratio between arterial $\mathrm{pO}_{2}$ and $\mathrm{FiO}_{2}$, was calculated and reported as well.

Information about ventilation, in particular noninvasive ventilation (NIV) and invasive ventilation by endotracheal tube (ET), were recorded.

We registered information about overall hospitalization time, in particular, dividing patients according to the addressing ward (non-intensive ward and intensive-care unit), time-to-discharge, and time-to-death in the period between March 4, and June 4, 2020.

\section{CTPA technique}

All CTPA examinations were performed using a 256-slice scanner (iCT Elite-Philips, Eindhoven), during contrast media injection (50 ml, Iobitridol 350-Xenetix 350 Guerbet, France; flow rate $3.5 \mathrm{ml} / \mathrm{s}$ ) followed by saline flush ( $50 \mathrm{ml}$, flow rate $3.5 \mathrm{ml} / \mathrm{s}$ ) by using bolus tracking technique, with a trigger level of 120 Hounsfield unit (HU) and a delay time of $8 \mathrm{~s}$. The trigger area was manually placed in the main pulmonary artery. The parameters for CTPA scans were tube voltage $80 \mathrm{kV}$, automated tube current modulation, collimation $128 \times 0.625$, pitch 0.617 , rotation time $0.27 \mathrm{~s}$, field of view (FOV) 350, matrix $512 \times 512$, thickness $0.8 \mathrm{~mm}$, and increment $0.4 \mathrm{~mm}$. CT images were acquired during a single breath-hold. Multiplanar reconstruction on the sagittal and the coronal plane, and the maximum intensity projection (MIP) reconstruction images were obtained.

\section{Image analysis}

Image analysis was performed using the institutional digital database system PACS (Enterprise imaging, AGFA Healthcare, Belgium) by a radiologist with 15 years of experience in chest imaging and a resident radiologist with 4 years of experience, who independently reviewed the images. Final scores were determined by consensus. For each patient, the CTPA scan evaluation included: (1) main, right, and left pulmonary artery (MPA, RPA, LPA) diameters, (2) right ventricle/left ventricle ( $\mathrm{RV} / \mathrm{LV})$ ratio, (3) presence of $\mathrm{PE}$, (4) pulmonary artery obstructive index (PAO Index), (5) PE distribution, central o peripheral, (s6) the temporal stage of lung disease and (7) the extent of lung involvement.

The RV/LV ratio was calculated from the value of the minor axes of heart right and left ventricles, measured in the transverse plane at their widest points between the inner surface of the free wall and the surface of the interventricular septum. An RV/LV ratio lesser than 1.0 was considered normal.

The PAO index was calculated according to Qanadli et al. [20], as the product of $N^{*} D$, where $N$ is the value of the proximal clot site equal to the number of segmental branches arising distally, and $D$ is the degree of obstruction, defined as 1 for non-occlusive vessels $(D 1)$ and 2 for totally occluded vessels (D2). Thus, an occluded embolus in a segmental artery was assigned a value of 2 . A clot in a proximal artery that gives rise to segmental arteries was scored as though each segmental artery had an embolus with the same degree of obstruction as the proximal embolus. Subsegmental emboli were scored as a partial obstruction of a segmental artery. The maximum obstruction score value was 40 and then converted into a percentage.

The temporal CT changes which indicate the disease progression was evaluated using the temporal CT staging system according to recent literature findings reported by Pan et al. [21]: early stage (0-4 days) as normal CT or ground-glass opacities (GGO); progressive stage (5-8 days) as increased GGO and crazy-paving pattern; peak stage (9-13 days) as consolidation; absorption stage (>14 days) as fibrous stripes.

Moreover, the lung CT involvement was assessed using a semiquantitative CT-severity score, previously reported by Liu et al. [22]: each of the five lung lobes was visually scored from 0 to 5 as follows: 0 ) no involvement, (1) less than 5\% involvement, (2) 6-25\% involvement, (3) 26-49\% involvement, (4) 50-75\% involvement, or (5) more than $75 \%$ involvement. The total CT score was the sum of the five individual lobar scores and ranged from 0 (no involvement) to 25 (maximum involvement).

\section{Statistical analysis}

Continuous variables were expressed as means and standard deviations and compared by using the Mann-Whitney test. Categorical variables were expressed as median value and interquartile range (IQR) and compared by using the Chi-square test or Fisher exact test, as appropriate. Relative risk (RR) and odds ratios (OR) were computed using crosstabs, with a $95 \%$ confidence interval (CI) as reference. A RR value of 1 implies that the presence of that variable poses no additional risk, RR greater than 1 or less than 1 implies additional or reduction in risk, respectively. OR greater than 1 represents an association between two variables. Correlations were computed with the Pearson or Spearman correlation coefficients, as appropriate. All tests were two-sided, and $p<0.05$ was considered statistically significant. All statistical analyses were performed using the SPSS statistical package software (SPSS v26.0-Chicago). 


\section{Results}

\section{Demographic, clinical, and laboratory data of the entire cohort}

By applying inclusion and exclusion criteria, a total of 170 patients were enrolled ( $n=24$ from Center $1, n=30$ from Center 2 , and $n=116$ from Center 3 ). The majority of patients were male, with a mean age of 63 years $( \pm 12)$, with no major risk factors nor a history of PE $(n=152,89.4 \%)$. All laboratory data are summarized in Table 1.

\section{Imaging findings}

The majority of patients were negative for PE at CT scan $(n=94,55.3 \%)$. Patients with a CT scan positive for PE $(44.7 \%)$ were more frequently male $(M / F=58 / 18, p=$ $0.061)$ with a mean age of 63 years $( \pm 14)$. The majority of PE-positive patients showed a peripheral $(n=44,57.9 \%)$ and bilateral ( $n=42,55.3 \%)$ distribution of the embolic event (Figs. 1 and 2), and a higher number of RV/LV enlargement $(n=68,89.5 \%, p<0.0001, \mathrm{OR}=71.40)$.

Overall, the mean MPA diameter was $27.17 \mathrm{~mm}( \pm 3.42)$, RPA $21.87 \mathrm{~mm}( \pm 3.82)$, LPA $21.57( \pm 3.47)$, and RV/LV ratio $0.99( \pm 0.18)$. The mean RPA and LPA diameters and $\mathrm{RV} / \mathrm{LV}$ ratio were significantly higher in the PE-positive group ( $p=0.039, p=0.006$, and $p<0.0001$, respectively). No significant difference was found in the MPA diameter between PE-positive and negative groups $(p=0.238)$. According to laboratory data, only $D$-Dimer showed a statistically significant difference between the two groups $(p=0.019)$, with a higher value in those patients positive for PE.

\section{PAO index}

The median value of the PAO index was 12.5 (2.5-50.0). Only six patients $(7.9 \%)$ presented a PAO index $>40 \%$.

Table $1 \mathrm{CT}$ and laboratory findings, medical and ventilation therapy in the cohort and between the two groups, according to the presence of PE. $p$ values in bold denote a significant difference

\begin{tabular}{|c|c|c|c|c|}
\hline$N=170$ & All & PE positive $(n=76)$ & PE negative $(n=94)$ & $p$ value \\
\hline Age $($ yo $\pm S D)$ & $63 \pm 12$ & $62 \pm 15$ & $60 \pm 14$ & 0.519 \\
\hline Sex male $(\mathrm{n} ; \%)$ & $116 ; 68.2$ & $58 ; 76.31$ & $58 ; 61.70$ & 0.061 \\
\hline $\mathrm{MPA}(\mathrm{mm} \pm \mathrm{SD})$ & $27.17 \pm 3.42$ & $27.74 \pm 3.79$ & $26.72 \pm 3.06$ & 0.238 \\
\hline $\mathrm{RPA}(\mathrm{mm} \pm \mathrm{SD})$ & $21.87 \pm 3.82$ & $22.63 \pm 3.54$ & $21.26 \pm 2.96$ & 0.039 \\
\hline $\mathrm{LPA}(\mathrm{mm} \pm \mathrm{SD})$ & $21.57 \pm 3.47$ & $22.68 \pm 3.43$ & $20.68 \pm 3.29$ & 0.006 \\
\hline $\mathrm{RV} / \mathrm{LV}$ ratio $( \pm \mathrm{SD})$ & $0.99 \pm 0.18$ & $1.14 \pm 0.17$ & $0.87 \pm 0.11$ & $<0.0001$ \\
\hline$D$-Dimer $(\mu \mathrm{g} / \mathrm{ml} \pm \mathrm{SD})$ & $6352 \pm 12,362.41$ & $32,231.26 \pm 5223.97$ & $10,051.80 \pm 17,289.01$ & 0.019 \\
\hline $\mathrm{WBC}\left(\times 10^{3} / \mathrm{mm}^{3} \pm \mathrm{SD}\right)$ & $8.71 \pm 4.06$ & $9.75 \pm 4.29$ & $8.08 \pm 3.85$ & 0.114 \\
\hline Neutrophils $\left(\times 10^{3} / \mathrm{mm}^{3} \pm \mathrm{SD}\right)$ & $7.07 \pm 3.65$ & $7.81 \pm 4.18$ & $6.71 \pm 3.39$ & 0.492 \\
\hline Lymphocytes $\left(\times 10^{3} / \mathrm{mm}^{3} \pm \mathrm{SD}\right)$ & $1.21 \pm 0.91$ & $1.51 \pm 1.37$ & $1.07 \pm 0.54$ & 0.806 \\
\hline $\operatorname{PLT}\left(\times 10^{3} / \mathrm{mm}^{3} \pm \mathrm{SD}\right)$ & $266.66 \pm 128.82$ & $270.42 \pm 126.82$ & $254.21 \pm 132.29$ & 0.621 \\
\hline $\mathrm{CRP}(\mathrm{mg} / \mathrm{l} \pm \mathrm{SD})$ & $10.38 \pm 11.75$ & $14.63 \pm 14.74$ & $14.68 \pm 13.38$ & 0.582 \\
\hline Fibrinogen $(\mathrm{mg} / \mathrm{dl} \pm \mathrm{SD})$ & $586.06 \pm 195.41$ & $620.18 \pm 195.13$ & $558.36 \pm 194.82$ & 0.325 \\
\hline ATIII $(\% \pm \mathrm{SD})$ & $88.39 \pm 19.39$ & $85.17 \pm 20.55$ & $94.20 \pm 16.53$ & 0.089 \\
\hline INR $( \pm$ SD $)$ & $1.24 \pm 0.51$ & $1.19 \pm 0.28$ & $1.29 \pm 0.67$ & 0.934 \\
\hline $\mathrm{SaO}_{2}(\%)$ & $92.96 \pm 7.90$ & $94 \pm 8$ & $92 \pm 8$ & 0.256 \\
\hline $\mathrm{pO}_{2}(\mathrm{mmHg} \pm \mathrm{SD})$ & $95.15 \pm 55.45$ & $91.4 \pm 49.5$ & $97.4 \pm 59.6$ & 0.930 \\
\hline $\mathrm{pCO}_{2}(\mathrm{mmHg} \pm \mathrm{SD})$ & $37.99 \pm 14.01$ & $40.0 \pm 20.6$ & $36.7 \pm 8.0$ & 0.807 \\
\hline $\mathrm{HCO}_{3}^{-}(\mathrm{mEq} / 1 \pm \mathrm{SD})$ & $24.68 \pm 5.71$ & $24.9 \pm 4.0$ & $24.4 \pm 6.6$ & 0.902 \\
\hline $\mathrm{pH}( \pm \mathrm{SD})$ & $7.0964 \pm 1.5213$ & $7.02 \pm 1.72$ & $7.15 \pm 1.41$ & 0.243 \\
\hline $\mathrm{P} / \mathrm{F}$ ratio $( \pm \mathrm{SD})$ & $220.91 \pm 140.56$ & $217.52 \pm 165.50$ & $233.49 \pm 122.50$ & 0.774 \\
\hline CT-staging system (median; IQR) & $12.5 ; 7-15.25$ & $14 ; 9-17$ & $11 ; 7-14$ & 0.103 \\
\hline Medical therapy (n; \%) & $68 ; 40.0$ & $32 ; 18.9$ & $36 ; 21.2$ & 0.518 \\
\hline NIV (n; \%) & $76 ; 44.7$ & $34 ; 20.0$ & $42 ; 24.7$ & 0.520 \\
\hline ET (n; \%) & $26 ; 15.3$ & $10 ; 5.9$ & $16 ; 9.4$ & 0.400 \\
\hline
\end{tabular}

$M P A$, main pulmonary artery; $R P A$, right pulmonary artery, $L P A$, left pulmonary artery; $R V / L V$, right ventricle/left ventricle ratio; $W B C$, white blood cell; PLT, platelets; CRP, C-reactive protein; ATIII, antithrombin III; INR, international normalized ratio; NIV, noninvasive ventilation; $E T$, endotracheal tube 

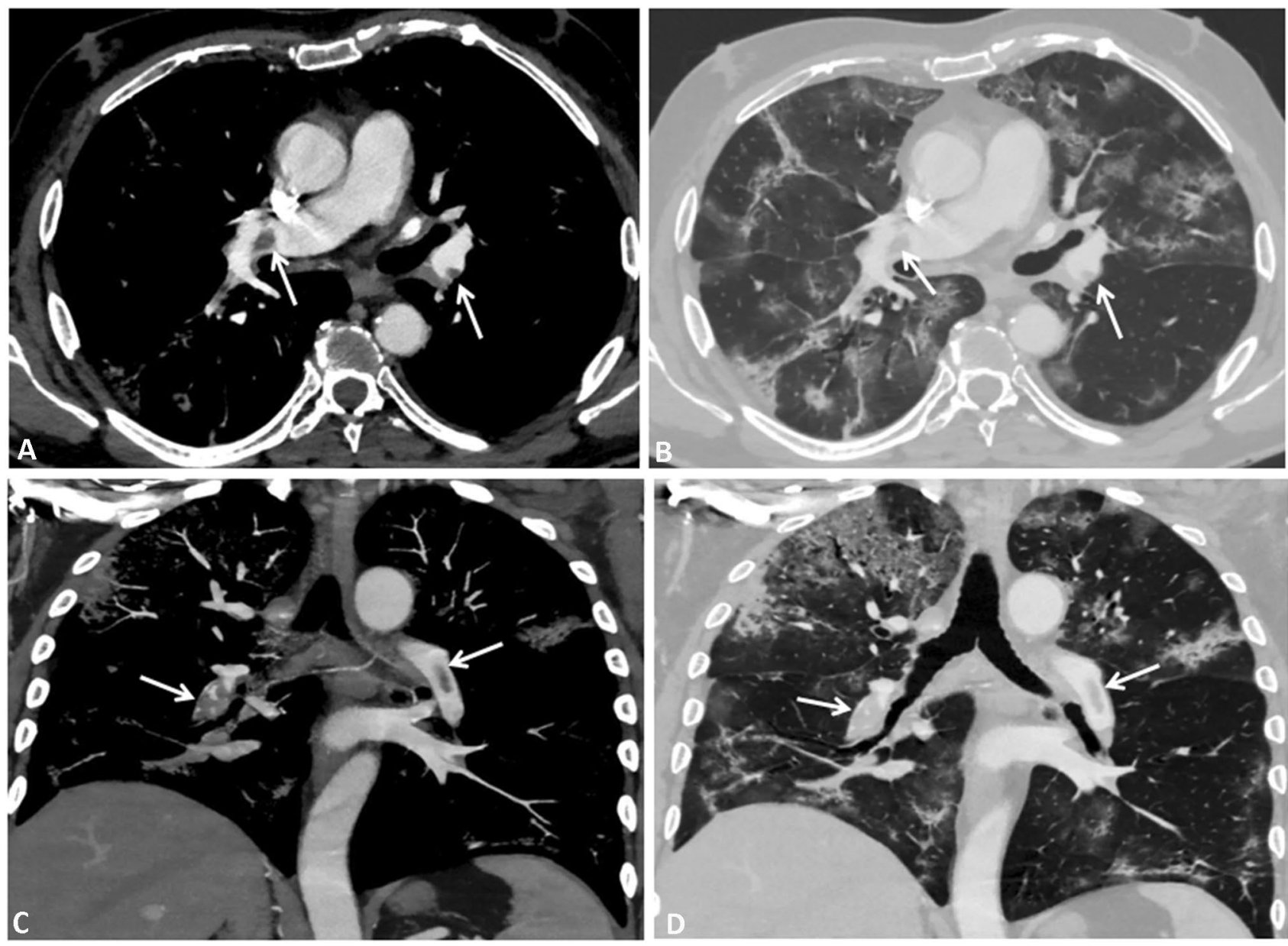

Fig. 1 CTPA images on the axial planes $\mathbf{a}, \mathbf{b}$ and MPR $\mathbf{c}, \mathbf{d}-$ Temporal CT-staging system Stage 1. a window width and level for the evaluation of mediastinum allow to correctly evaluate the presence of clot (arrows) inside the RPA and in the lobar branch for the left lower lobe. The clot appears as a hypoattenuating finding inside the vessel. b window width and level for the evaluation of lung parenchyma allow us to correctly evaluate the presence of multiple GGOs, distributed bilaterally, especially in the subpleural space. Moreover, reticu-

According to laboratory data, significant correlations with $D$-Dimer $(r=0.584, \mathrm{p}=0.002)$,

$\mathrm{CRP}(r=0.384, p=0.013), \mathrm{pH}(\mathrm{r}=0.339, p=0.029)$ and $P / F$ ratio $(r=-0.463, p=0.031)$ values were found.

\section{Temporal CT staging system}

No significant difference according to the temporal CT-staging system, between the two groups, was found $(p=0.273)$.

CT scans, both negative and positive for PE, were more frequently characterized by a score of $3(n=54,20.0 \%$ and $n=32,18.8 \%$ respectively). The presence of PE had an $\mathrm{OR}=2.26$ to obtain a score $\geq 3$ and an $\mathrm{OR}=2.12$ for a score equal to 4 . lations are diffuse bilaterally. c MPR image with window width and level for the evaluation of mediastinum confirms the presence of the clot and its extent. The clot inside the RPA lumen is considered completely occlusive, while in the left side sub-occlusive. d MPR image with window width and level for the evaluation of lung parenchyma confirms the presence of GGOs, especially in both upper lobes in the subpleural spaces. No lung effusion was present

Positive correlations between temporal CT-staging system and MPA $(r=0.229, p=0.018)$, RPA $(r=0.187$, $p=0.047)$, LPA $(r=0.186, r=0.045)$ diameters, $\mathrm{RV} / \mathrm{LV}$ ratio $(r=0.415, p<0.0001)$, were found.

Moreover, a positive correlation between the temporal CT-staging system and the PAO index was found $(r=0.291$, $p=0.036$ ) (Figs. 3 and 4).

\section{CT-severity Score}

The overall median value of the CT-severity score was 12.5 (IQR 7-15.25). No significant difference regarding CT-severity score values was obtained between the two groups [median value $11(7-14)$ and 14 (9-17) respectively, $p=0.103]$. 

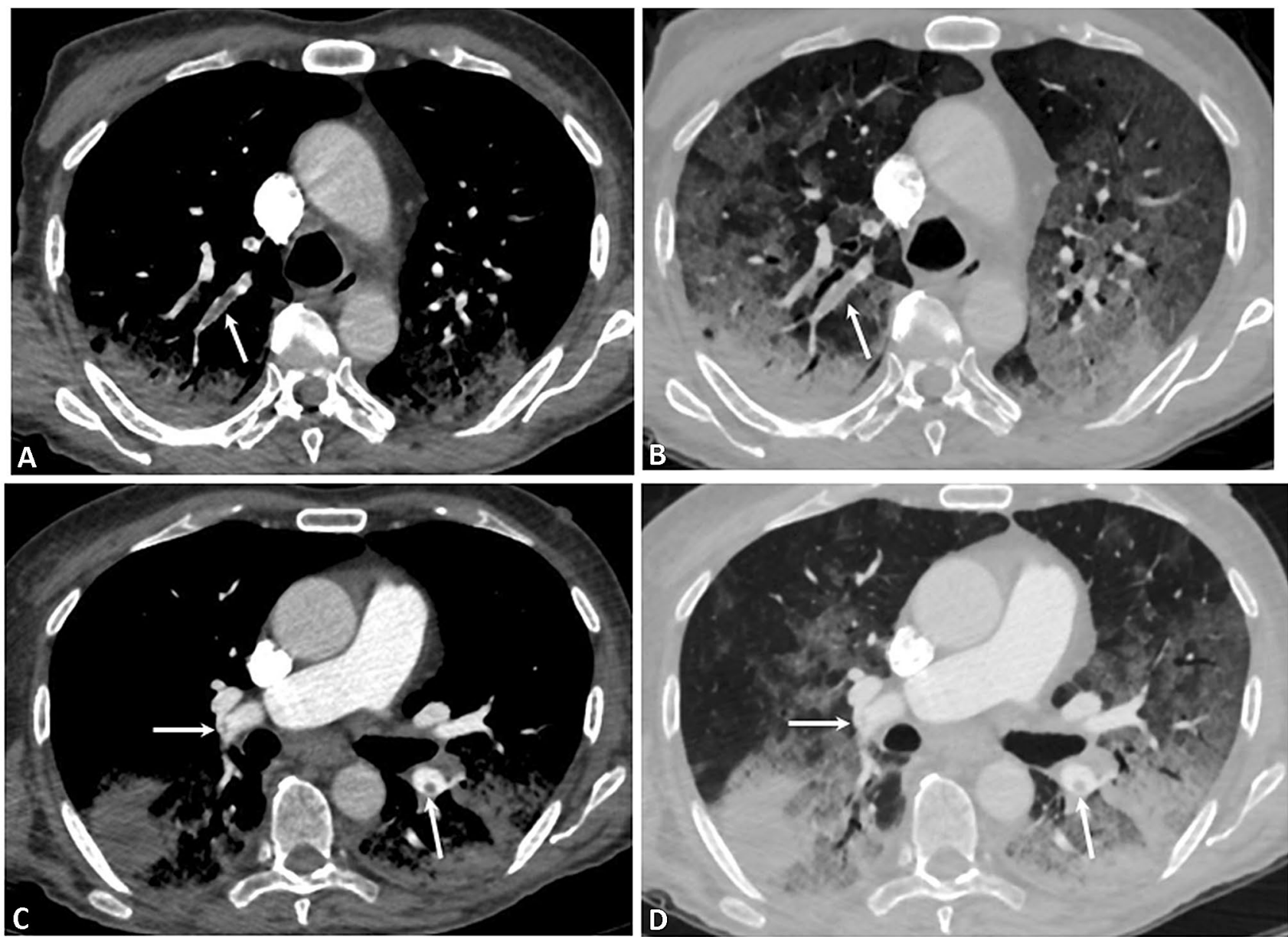

Fig. 2 CTPA images on the axial planes-CT-staging system Stage 3. a window width and level for the evaluation of mediastinum allow us to correctly evaluate the presence of clot (arrows) inside the lobar branch for the right upper lobe. $\mathbf{b}$ window width and level for the evaluation of lung parenchyma allow us to correctly evaluate the presence of diffuse and confluent GGOs with air bronchogram associated. $\mathbf{c}$ window width and level for the evaluation of mediasti-

Significant correlations between the CT-severity score and $D$-Dimer $(\mathrm{r}=0.615, \mathrm{p}=0.001)$, fibrinogen $(r=0.317$, $p=0.015)$, ATIII $(r=-0.572, p=0.001), \mathrm{pCO}_{2}(r=0.199$, $p=0.050), \mathrm{HCO}_{3}{ }^{-}(r=0.220, p=0.039)$, and $\mathrm{FiO}_{2}(r=0.436$, $p=0.001)$, were found

\section{Ventilation}

Overall, 68 patients (40.0\%) underwent medical therapy while $102(60.0 \%)$ ventilation, of whom $76(74.5 \%)$ NIV and $26(25.5 \%)$ with ET. From a total of 76 PE-positive patients, $32(42.1 \%)$ underwent medical therapy, while $44(57.9 \%)$ ventilation $[n=34(44.7 \%) \mathrm{NIV}$, and $n=10(13.2 \%)$ invasive ventilation by ET]. The median PAO index value was significantly higher in patients who underwent ventilation, both NIV and ET $(p=0.010)$. According to laboratory data,

num allow us to correctly evaluate the presence of small clot (arrow) inside the lobar branch of RPA. d window width and level for the evaluation of lung parenchyma allow us to correctly evaluate the presence of diffuse and confluent GGOs with air bronchogram associated. Moreover, it is possible to appreciate the presence of bilateral consolidations, with a subpleural distribution

CRP values and $P / F$ ratio showed significant differences in patients who underwent ventilation $(p=0.002$ and $p=0.013$ respectively) (supplementary table).

The temporal CT-staging system between patients who underwent ventilation and those who did notshowed a statistically significant difference, both in terms of NIV and ET $(p=0.004)$, in particular for patients included in classes 3 and $4(p<0.0001)$. CT-severity score values were significantly higher in patients who underwent ventilation $(p=0.007)$, but without a significant difference in terms of hospitalization ward $(p=0.277)$.

\section{Hospitalization and outcome}

From a total of 76 PE-positive patients, 66 (87\%) were hospitalized in non-intensive wards while only $10(13 \%)$ were 


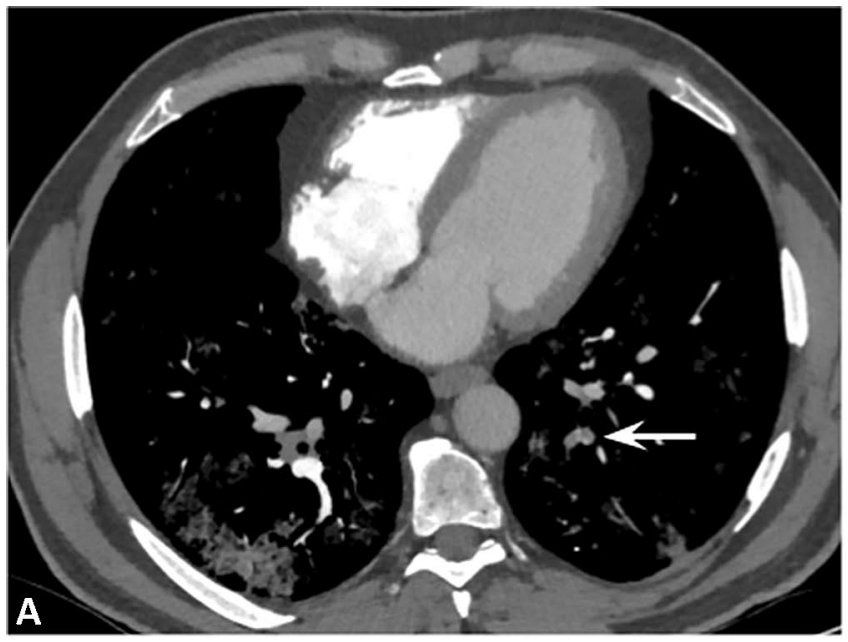

Fig. 3 CTPA images on the axial planes. CT-staging system Stage 4. a window width and level for the evaluation of mediastinum allow us to correctly evaluate the presence of clot (arrows) inside the lobar branch for the left lower lobe. $\mathbf{b}$ window width and level for the eval-

hospitalized in ICUs. PE-positive patients showed a slightly higher hospitalization time in comparison with PE-negative ones [mean $30( \pm 18)$ vs $24( \pm 19)$, median $26(17-46)$ vs. 19 (7-40) days respectively], without statistically significant difference $(p=0.177)$. Moreover, hospitalization time did not show a statistically significant difference between ICU and non-intensive wards [mean $29( \pm 22)$ vs. $26( \pm 18)$, median 19 (15-36) vs. 22 (12-42) days respectively, $p=0.668$ ] (supplementary table). In the 3-month period of study, 146 patients $(85.9 \%)$ were discharged, while 24 patients $(14.1 \%)$ died, of whom 13 PE-positive (54.2\%) and 9 PE-negative $(45.8 \%)(p=0.365, \mathrm{OR}=1.54-95 \%$ CI $0.430-5.495)$. No statistically significant differences among all CT findings nor laboratory data and patient's death were found (all $p>0.05$ ).

\section{Discussion}

Along with other established risk factors, the presence of acute infections in both hospitalized and non-hospitalized patients is deeply associated with the increased risk of venous thromboembolism, which includes deep vein thrombosis and PE [21, 22].

The increased risk of PE after acute infection, especially the respiratory tract, might be caused by local inflammation leading to local activation of coagulation and local vasoconstriction induced by the hypoxic lung environment in the infected area [23].

Therefore, accurate knowledge of thrombotic complications, such as acute PE, in patients infected by SARS-CoV-2 is crucial for monitoring disease evolution and depicting worsening or other complications.

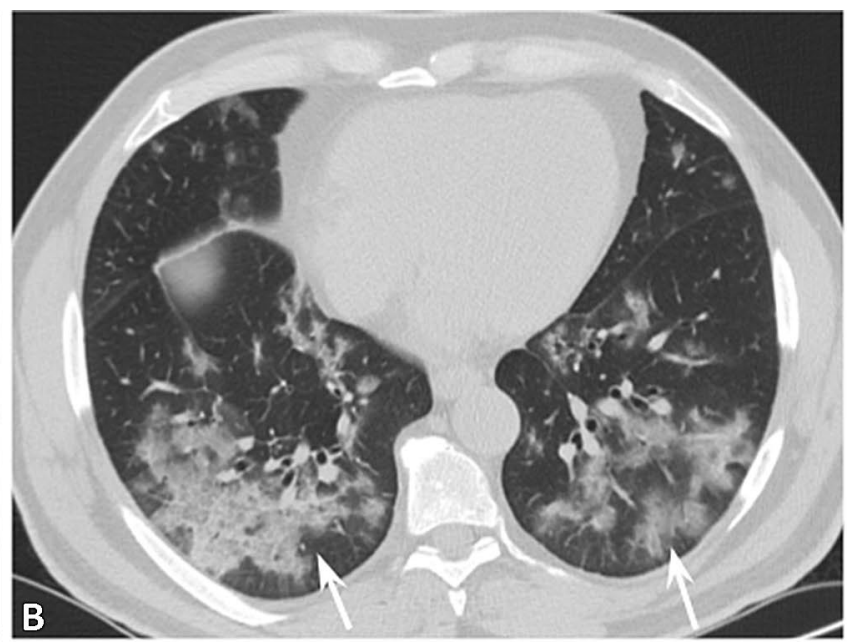

uation of lung parenchyma allow us to correctly evaluate the presence of diffuse GGOs, in particular in the subpleural space of the left lung, associated with more evident reticulations. In the right lower lobe, it is possible to appreciate the presence of a consolidation

Among all patients enrolled in our study, $44.7 \%$ developed pulmonary embolism during hospitalization despite any major risk factors for venous thromboembolism and having undergone, according to their $D$-dimer score, either prophylactic or complete anticoagulation therapy before CTPA examination, according to the guidelines implemented by the hospitals taking part in the study. The causal relationship between PE and severe infection in our cohort is made clear by the absence of predisposing thrombo-embolic factors.

Some authors supported the existence of a significant correlation between SARS-CoV-2 and PE due to endothelial dysfunction and to a hypercoagulation state with the activation of platelets and leukocytes which result in thrombin generation and widespread fibrin deposition $[17,18]$. This condition is further aggravated by hypoxia, which augments thrombosis by both increasing blood viscosity and hypoxiainducible transcription factor-dependent signaling pathway [23-25].

More specifically, a recent study found a pro-coagulation pattern characterized by increased clot strength in SARS$\mathrm{CoV}-2$ patients, which significantly responded to thromboprophylaxis [25].

However, the reason for PE occurrence in SARS-CoV-2 patients is still unclear.

In our series, the most diffuse pulmonary arteries involvement occurred in those patients with markedly elevated $D$-dimer and CRP values, despite the high dose anticoagulation regimen, and in those patients with a low value of $\mathrm{P} / \mathrm{F}$. These results suggested that SARS-CoV-2 patients with higher levels of inflammation and higher values of $D$-dimer are more prone to develop pulmonary embolism underlining the importance of monitoring these biochemical values 

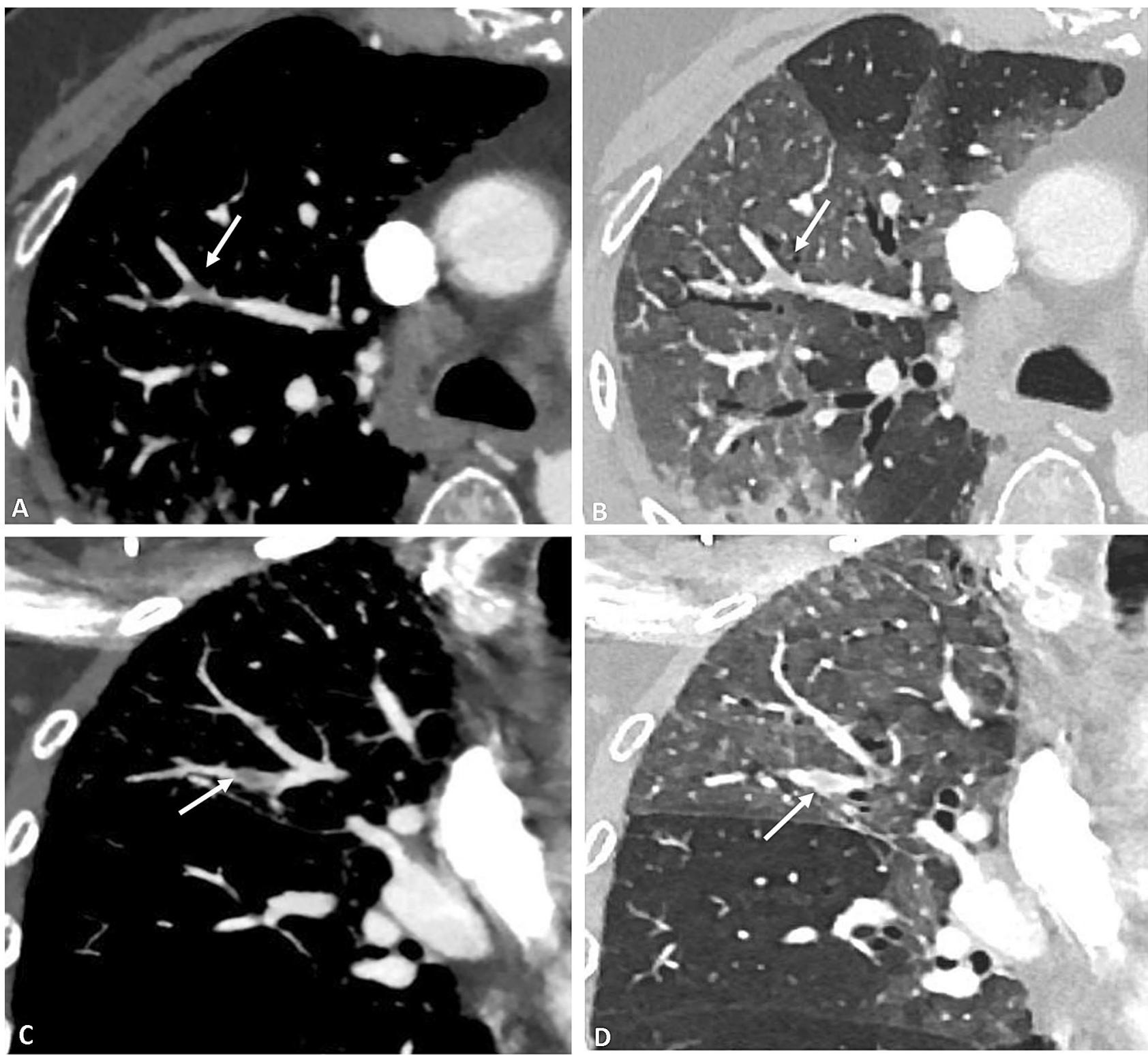

Fig. 4 CTPA images on the axial planes $(\mathbf{a}, \mathbf{b})$, and coronal plane (c, d) - CT-staging system Stage 2. (a, c) Window width and level for the evaluation of mediastinum allow us to correctly evaluate the presence of clot (arrows) inside the segmental and subsegmental branches of

the RUL. (b, d) Window width and level for the evaluation of lung parenchyma allow us to correctly evaluate the presence of multiple and confluent GGOs in the RUL. (c)

to promptly identify the patients at higher risk of PE, and quickly perform CTPA.

Although our cohort did not show a direct correlation between the development of an embolic event and the CT staging system, our results showed an increment of the occurrence of a severe thromboembolic event, according to PAO index values, in the advanced stages of lung involvement Interestingly, from pathophysiologic view, the positive correlations between temporal CT-staging system and MPA $(r=0.229, p=0.018)$, RPA $(r=0.187, p=0.047)$, LPA $(r=0.186, r=0.045)$ diameters, $\mathrm{RV} / \mathrm{LV}$ ratio

$(r=0.415, p<0.0001)$, indicate the increased resistance of pressure of the arterial pulmonary flow. These results demonstrate that SARS-CoV-2 infection, in combination with mechanical ventilation, can cause an increased RV afterload, with the consequent possibility to develop PE.

As for the CT-severity score, in line with the result of the recent paper of Gervaise et al. [26], our study did not show any significant difference concerning the extent of lung involvement in COVID-19 patients with or without PE. This supports the evidence that the increased risk of 
developing pulmonary embolism is not directly correlated to the severity of lung disease.

In our series, most of the registered thromboembolic events were bilateral and non-massive with a median PAO index value of 12.50 . Only 6 out of 76 patients (7.8\%) presented a PAO Index $>40 \%$, indicating a massive embolic event. A slight majority of patients (57.9\%) developed a peripheral segmental or subsegmental embolism.

Although we did not find a significant difference in terms of therapy, both medical and mechanical ventilation (noninvasive ventilation and endotracheal intubation), between patients who developed PE and those did not, our results indicates that patients with higher PAO Index values underwent more frequently ventilation, both NIV and ET. This may suggest that patients who developed a more severe embolic event are more prone to be hospitalized in ICU which underlines again the importance to evaluate the presence of pulmonary embolism and in particular the extent of the embolic event.

Furthermore, invasive and noninvasive ventilation was frequently found in association with the more advanced stages of parenchymal involvement.

Moreover, our results highlight that PE-positive patients presented the worst general health condition as they needed a slightly longer recovery than patients without PE.

In line with a recent paper [27], we did not find a significant difference in the ICU admission and the need for intubation between the two groups (PE positive and PE negative).

Indeed $87 \%$ of patients positive for pulmonary embolism were not hospitalized in ICU suggesting that every patient affected by COVID-19, even if they do not have severe health conditions to qualify for ICU care, can develop an acute pulmonary embolism.

A low mortality incidence of patients with PE and SARSCoV-2-related pneumonia was registered in our series, maybe because of the prompt and rapid anticoagulant administration, and the strict observation regimen in those hospitalized patients.

In our series, we confirmed that PE should be considered a common complication in patients with SARS-CoV2-related pneumonia, in line with recently published studies $[13,28,29]$.

This study presents some limitations. Firstly, its retrospective nature. Secondly, the CTPAs were performed only in those patients with clinical and laboratory data suspicious for PE, and therefore the overall correct incidence and prevalence of PE in SARS-CoV-2 patients cannot be determined. Thirdly, CTPAs were usually performed when most of the hospitalized patients already presented advanced pneumonia, thus making an evaluation of the association between $\mathrm{PE}$ and CT-stage system difficult.

In conclusion, patients hospitalized for SARS-CoV-2 infection present a higher cumulative incidence of $\mathrm{PE}$ compared to the general population of hospitalized patients, regardless of the severity of lung inflammation or the temporal stage of the disease.

Therefore, it is required constant monitoring of $D$-dimer and CRP values along with the support of CTPA, for the evaluation of both parenchymal involvement and pulmonary artery obstruction, useful to address the correct clinical management of patients.

Funding No funding needed.

\section{Compliance with ethical standards}

Conflict of interest The authors declares that they have no conflict of interest.

Consent to participate All patients signed the informed consent form to be eligible for this study.

Consent for publication All patients gave the consent for information about themselves to be published in scientific journals.

Data availability and materials All data generated or analyzed during this study are included in this published article.

Ethical approval Local Ethical Committee's review of the protocol deemed that formal approval was not required owing to the retrospective, observational, and anonymous nature of this study.

\section{References}

1. World Health Organization (2019) Statement on the second meeting of the International Health Regulations. Emergency Committee regarding the outbreak of novel coronavirus. www.who.int/ news-room

2. Huang C, Wang Y, Li X et al (2020) Clinical features of patients infected with 2019 novel coronavirus in Wuhan, China. The Lancet 395:497-506. https://doi.org/10.1016/S0140-6736(20)30183 $-5$

3. Shi $\mathrm{H}$, Han $\mathrm{X}$, Jiang $\mathrm{N}$ et al (2020) Radiological findings from 81 patients with COVID-19 pneumonia in Wuhan, China: a descriptive study. Lancet Infect Dis 20:425-434. https://doi.org/10.1016/ S1473-3099(20)30086-4

4. China National Health Commission (2020) Notice on issuing a new coronavirus infected pneumonia diagnosis and treatment plan (trial version 5). bgs.satcm.gov.cn/ zhengcewenjian/2020-02-06/12847

5. Revel M-P, Parkar AP et al (2020) COVID-19 patients and the radiology department-advice from the european society of radiology (ESR) and the european society of thoracic imaging (ESTI). Eur Radiol. https://doi.org/10.1007/s00330-020-06865-y

6. Rubin GD, Ryerson CJ, Haramati LB et al (2020) The role of chest imaging in patient management during the COVID-19 pandemic: a multinational consensus statement from the fleischner society. Radiology 296:172-180. https://doi.org/10.1148/radiol.20202 01365

7. Wang D, Hu B, Hu C et al (2020) Clinical characteristics of 138 hospitalized patients with 2019 novel coronavirus-infected 
pneumonia in Wuhan. China JAMA 323:1061. https://doi. org/10.1001/jama.2020.1585

8. Lee EYP, Ng M-Y, Khong P-L (2020) COVID-19 pneumonia: what has CT taught us? Lancet Infect Dis 20:384-385. https:// doi.org/10.1016/S1473-3099(20)30134-1

9. Zhao W, Zhong Z, Xie X et al (2020) Relation between chest ct findings and clinical conditions of coronavirus disease (COVID19) pneumonia: a multicenter study. Am J Roentgenol 214:10721077. https://doi.org/10.2214/AJR.20.22976

10. Kanne JP, Little BP, Chung JH et al (2020) Essentials for radiologists on COVID-19: an update-radiology scientific expert panel. Radiology. https://doi.org/10.1148/radiol.2020200527

11. Bompard F, Monnier H, Saab I et al (2020) Pulmonary embolism in patients with Covid-19 pneumonia. Eur Respir J. https://doi. org/10.1183/13993003.01365-2020

12. Leonard-Lorant I, Delabranche X, Severac F et al (2020) Acute pulmonary embolism in COVID-19 patients on CT angiography and relationship to D-Dimer levels. Radiology. https://doi. org/10.1148/radiol.2020201561

13. Grillet F, Behr J, Calame P et al (2020) Acute pulmonary embolism associated with COVID-19 pneumonia detected by pulmonary ct angiography. Radiology. https://doi.org/10.1148/radio 1.2020201544

14. Rodrigues JCL, Hare SS, Edey A et al (2020) An update on COVID-19 for the radiologist-a British society of Thoracic Imaging statement. Clin Radiol 75:323-325. https://doi. org/10.1016/j.crad.2020.03.003

15. Arachchillage DRJ, Laffan M (2020) Abnormal coagulation parameters are associated with poor prognosis in patients with novel coronavirus pneumonia. J Thromb Haemost 18:1233-1234. https://doi.org/10.1111/jth. 14820

16. Wu AS, Pezzullo JA, Cronan JJ et al (2004) CT Pulmonary angiography: quantification of pulmonary embolus as a predictor of patient outcome-initial experience. Radiology 230:831-835. https://doi.org/10.1148/radiol.2303030083

17. Schmidt M, Horvath-Puho E, Thomsen RW et al (2012) Acute infections and venous thromboembolism: Infections and VTE. J Intern Med 271:608-618. https://doi.org/10.111 1/j.1365-2796.2011.02473.x

18. Smeeth L, Cook C, Thomas S et al (2006) Risk of deep vein thrombosis and pulmonary embolism after acute infection in a community setting. The Lancet 367:1075-1079. https://doi. org/10.1016/S0140-6736(06)68474-2

19. Chen $\mathrm{T}, \mathrm{Wu} \mathrm{D}, \mathrm{Chen} \mathrm{H}$ et al (2020) Clinical characteristics of 113 deceased patients with coronavirus disease 2019: retrospective study. BMJ. https://doi.org/10.1136/bmj.m1091
20. Qanadli SD, El Hajjam M, Vieillard-Baron A et al (2001) New CT index to quantify arterial obstruction in pulmonary embolism: comparison with angiographic index and echocardiography. Am J Roentgenol 176:1415-1420. https://doi.org/10.2214/ ajr.176.6.1761415

21. Pan F, Ye T, Sun P et al (2020) Time course of lung changes at chest ct during recovery from coronavirus disease 2019 (COVID19). Radiology 295:715-721. https://doi.org/10.1148/radiol.20202 00370

22. Liu D, Li L, Wu X et al (2020) Pregnancy and perinatal outcomes of women with coronavirus disease (COVID-19) pneumonia: a preliminary analysis. Am J Roentgenol 215:127-132. https://doi. org/10.2214/AJR.20.23072

23. Gando S, Levi M, Toh C-H (2016) Disseminated intravascular coagulation. Nat Rev Dis Primers 2:16037. https://doi. org/10.1038/nrdp.2016.37

24. Marshall BE, Hanson CW, Frasch F, Marshall C (1994) Role of hypoxic pulmonary vasoconstriction in pulmonary gas exchange and blood flow distribution: 2. Pathophysiol Inten Care Med 20:379-389. https://doi.org/10.1007/BF01720916

25. Ranucci M, Ballotta A, Di Dedda U et al (2020) The procoagulant pattern of patients with COVID-19 acute respiratory distress syndrome. J Thromb Haemost 18:1747-1751. https://doi. org/10.1111/jth.14854

26. Gervaise A, Bouzad C, Peroux E, Helissey C (2020) Acute pulmonary embolism in non-hospitalized COVID-19 patients referred to CTPA by emergency department. Eur Radiol. https://doi. org/10.1007/s00330-020-06977-5

27. Poyiadji N, Cormier P, Patel PY (2020) Acute pulmonary embolism associated with covid-19 pneumonia detected with pulmonary ct angiography. Radiology. https://doi.org/10.1148/radio 1.2020201955

28. Danzi GB, Loffi M, Galeazzi G, Gherbesi E (2020) Acute pulmonary embolism and COVID-19 pneumonia: a random association? Eur Heart J 41:1858-1858. https://doi.org/10.1093/eurhe artj/ehaa254

29. Poissy J, Goutay J, Caplan M et al (2020) Pulmonary embolism in patients with covid-19: awareness of an increased prevalence. Circulation 142:184-186. https://doi.org/10.1161/CIRCULATIO NAHA.120.047

Publisher's Note Springer Nature remains neutral with regard to jurisdictional claims in published maps and institutional affiliations. 